(NASA-TM-111221) THERMOCAPILLARY

N96-17863

FLOW WITH EVAPORATION AND

CONDENSATION AT LOW GRAVITY. PART

2: DEFORMABLE SURFACE (NASA.

Marshall Space Flight (enter) $18 \mathrm{p}$

Unclas

$63 / 34 \quad 0098275$ 


\title{
Thermocapillary flow with evaporation and condensation at low gravity. Part 2. Deformable surface
}

\author{
By G. R. SCHMIDT ${ }^{1}$, T. J. CHUNG ${ }^{2}$ AND A. NADARAJAH ${ }^{3}$ \\ ${ }^{1}$ Propulsion Laboratory, NASA Marshall Space Flight Center, Huntsville, AL 35812, USA \\ ${ }^{2}$ Department of Mechanical Engineering, University of Alabama in Huntsville, Huntsville, \\ AL 35899, USA \\ ${ }^{3}$ Department of Chemical Engineering, University of Alabama in Huntsville, Huntsville, \\ AL 35899, USA
}

(Received 30 July 1992 and in revised form 2 February 1995)

The free surface behaviour of a volatile wetting liquid at low gravity is studied using scaling and numerical techniques. An open cavity model, which was applied in part 1 to investigate fluid flow and heat transfer in non-deforming pores, is used to evaluate the influence of convection on surface morphology with length scales and subcooling/superheating limits of $1 \leqslant D \leqslant 10^{2} \mu \mathrm{m}$ and $\sim 1 \mathrm{~K}$, respectively. Results show that the menisci shapes of highly wetting fluids are sensitive to thermocapillary flow and to a lesser extent the recoil force associated with evaporation and condensation. With subcooling, thermocapillarity produces a suction about the pore centreline that promotes loss of mechanical equilibrium, while condensation exerts an opposing force that under some conditions offsets this destabilizing influence. With superheating, thermocapillarity and evaporation act in the same direction and mutually foster surface stability. All of these trends are magnified by high capillary and Biot numbers, and the stronger circulation intensities associated with small contact angles. These phenomena strongly depend on the thermal and interfacial equilibrium between the liquid and vapour, and have important ramifications for systems designed to maintain a pressure differential across a porous surface.

\section{Introduction}

Several engineering applications exploit the capillary-dominant environment of porous surfaces to segregate liquids from either vapours or gases. The liquid/vapour interface typically consists of numerous small menisci which attach to a geometrically complex wicking structure and sustain a pressure difference between the two phases. The maintenance of this pressure differential and mechanical equilibrium for the meniscus within each surface pore is referred to as 'fluid retention' and is crucial for proper functioning of these systems.

In part 1 of this study (Schmidt, Chung \& Nadarajah 1995), we investigated the fluid mechanics and heat transfer in such pores. Although the problem domain used was similar to the float zones studied in materials processing, the features of liquid/vapour phase change, small contact angles and liquid exchange with a large reservoir made this problem unique and in some ways more complex than previous float-zone assessments. The numerous unknowns associated with coupled solution of the flow field, temperature and free surface geometry prompted us to first analyse the problem assuming a non-deforming meniscus. This was equivalent to presuming a vanishingly 
small ratio between viscous and surface tension forces (i.e. low capillary number $C a$ ) and was consistent with the length scales considered in materials processing applications. Even with this simplification, we found the flow and temperature fields to be quite different than those encountered in float-zone problems owing to the competition between thermocapillary and evaporation/condensation-induced circulation.

We also identified several reasons to suspect that convection could contribute to surface deformation and possibly loss of mechanical equilibrium. First, the cavity dimensions characteristic of porous surfaces are extremely small (i.e. $1 \leqslant D \leqslant 10^{2} \mu \mathrm{m}$ ) and yield larger $\mathrm{Ca}$ values than float-zone-type problems. Hence, the convection associated with thermocapillarity and liquid/vapour phase change could exert a strong influence on surface morphology. Secondly, the study showed that the cellular flow and small contact angles present in individual pores promote high dynamic pressure gradients near the meniscus contact line. This suggests that fixed meniscus shapes cannot be sustained under some conditions. Thus, a complete analysis of this problem requires that meniscus deformation be considered.

The complexity of including free surface deformation poses a serious challenge from an analytical standpoint. The computational resources necessary to perform a complete parametric study, as we did earlier for the fixed meniscus problem, are almost prohibitive. It is desirable to first perform a simplified analysis that identifies the parameter ranges where meniscus deformation becomes significant. A more exact analysis involving simultaneous solution of the flow field and surface at sensitive parameter values can then follow. In the next section, we present the modifications to the original theoretical model required to accommodate meniscus deformation. A description of the solution method used to perform both the preliminary and more exact analyses is given in $\S 3$, and is followed by a presentation and discussion of results in $\S \S 4$ and 5.

\section{Theoretical model}

To examine the influence of deformation, we employ the same problem domain, governing equations, boundary equations and assumptions that we used in part 1 (Schmidt et al. 1995). The domain is depicted in figure 1, and consists of a twodimensional rectangular groove partially filled with an incompressible Newtonian liquid. The cavity is assumed to be oriented with respect to a uniform vertical acceleration field. The left and right sides of the pore (boundaries 1 and 3 , respectively) consist of solid vertical sidewalls. The lower boundary 2 , however, is assumed to open to a large reservoir of liquid to enable balancing of mass flow through the cavity and approximate the performance of a capillary structure. Unlike in part 1 , the upper surface (boundary 4) is represented by a deformable meniscus which is symmetric about the pore centreline and bounded by an inert vapour. The shape of the interface is defined by the surface height above the base $y^{(s)}$ which is a function of the lateral coordinate $x_{1}$. The interface is assumed to wet the solid sidewalls at an acute contact angle $\omega$, and is further characterized by the unit normal and tangent vectors $\left(n_{i}\right.$ and $\left.s_{i}\right)$ and contour angle $\alpha$.

Another dependent variable is introduced by $y^{(s)}$ which must be coupled with solution of the flow parameters. We obtain an equation for $y^{(s)}$ from the normal component of the jump momentum balance along the meniscus. Ignoring viscous stress in the vapour, this simplifies to

$$
\left(P-\rho g y^{(\delta)}-P^{(v)}\right)+j^{2}\left(1 / \rho-1 / \rho^{(v)}\right)-2 \mu V_{i, j} n_{j} n_{i}+\gamma \kappa=0 .
$$




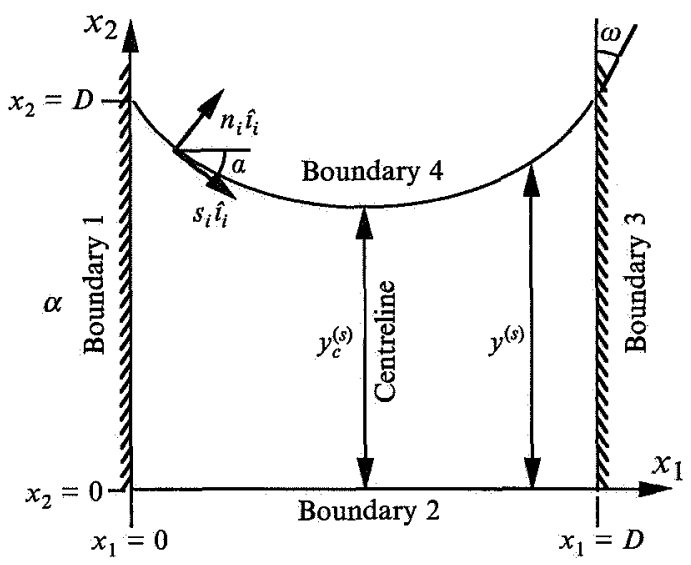

Figure 1. Problem domain.

The variables $V_{i}, P, g, \mu, \rho, \gamma$ and $\kappa$ are the velocity, dynamic pressure, gravitational acceleration, dynamic viscosity, density, surface tension and curvature, respectively. Note that the radius of curvature $1 / \kappa$ is considered positive when it extends into the vapour. The superscript $(v)$ refers to the variables in the vapour phase. The expression $P-\rho g y^{(s)}-P^{(v)}$ in (1) includes the hydrostatic contribution to the total pressure within the liquid; $j^{2}\left(1 / \rho-1 / \rho^{(v)}\right)$ represents the momentum change and recoil force arising from liquid evaporation or vapour condensation; $j$ is the mass flux across the surface and is obtained from the jump mass balance, that is

$$
j=\rho\left(V_{j}-V_{j}^{(s)}\right) n_{j}=\rho^{(v)}\left(V_{j}^{(v)}-V_{j}^{(s)}\right) n_{j},
$$

where $V_{j}^{(s)}$ is the velocity of the interface. Because $\rho>\rho^{(v)}$, the momentum flux on the vapour side of the surface is greater than that on the liquid side. Consequently, the recoil force exerted on the interface always points into the liquid, regardless of the direction of mass transfer. The third term, $2 \mu V_{i, j} n_{j} n_{i}$, reflects the effects of viscous stress, while the last term $\gamma \kappa$ represents the capillary pressure modification due to surface tension and curvature.

Using the same scaling methodology applied in part 1, (1) and (2) are nondimensionalized according to the pore width $D$, viscous time scale $D^{2} / \nu$ and maximum cavity temperature difference $|\Delta T|=\left|T_{1}-T_{0}\right|$. The variables $\nu, T_{1}$ and $T_{0}$ are the kinematic viscosity, sidewall temperature and vapour temperature, respectively. Equation (1) can be converted into an expression for curvature, which in dimensionless form becomes

$$
\kappa=\frac{B o y^{(s)}-C a\left(P-P^{(v)}\right)+V r T^{2}+2 C a V_{i, j} n_{j} n_{i}}{1-C r \bar{T}},
$$

where

$$
\begin{gathered}
B o=\rho g D^{2} / \gamma_{l}, \\
C r=|\partial \gamma / \partial T||\Delta T| / \gamma_{l}, \\
C a=\rho \nu^{2} /\left(\gamma_{l} D\right), \\
V r=C a\left(f_{\rho}-1\right) / R s^{2} .
\end{gathered}
$$

$\mathrm{Bo}, \mathrm{Cr}$ and $\mathrm{Ca}$ are the Bond, crispation and capillary numbers, respectively, while $\mathrm{Vr}$ is the recoil parameter. Here, $f_{\rho}$ is the ratio of liquid to vapour density $\rho / \rho^{(v)}, \gamma_{l}$ is the surface tension at the lowest temperature, and $R s$ is the interfacial resistance which was defined in part 1 . The terms comprising (3) are the different normal force contributions 


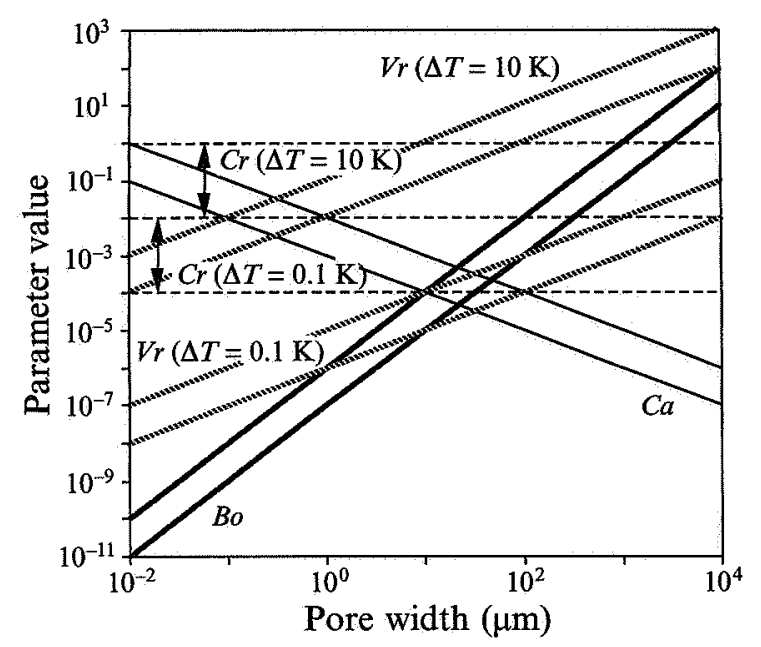

FrgURE 2. Sensitivity of dimensionless parameters associated with solution of $y^{(8)}$ : upper and lower bounds.

along the surface, where $B o y_{0}^{(s)}, C a\left(P-P^{(v)}\right), V r T^{2}, 2 C a V_{i, j} n_{j} n_{i}$ and $(1-C r \bar{T})^{-1}$ represent the influence of hydrostatic pressure, dynamic pressure, vapour recoil, viscous stress and surface tension thermal variation, respectively. $\bar{T}$ is a corrected scaled temperature whose value depends on the maximum and minimum temperature in the cavity. For superheating, $\bar{T}=T$, while for subcooling, $\bar{T}=T+1$. Note that $C a$, $\mathrm{Cr}$ and the Marangoni number $\mathrm{Ma}$ defined in our previous study are not mutually independent, since $\mathrm{Cr}$ can be expressed as $\mathrm{Cr}=\mathrm{MaCa} / \mathrm{Pr}$.

In addition to (3), $y^{(s)}$ must satisfy two boundary conditions at the sidewalls. These are the pinning condition and the contact angle constraint, and can be expressed as

$$
y^{(s)}=1 \text { and }\left|\mathrm{d} y^{(s)} / \mathrm{d} x_{1}\right|=\tan (\pi / 2-\omega) \quad \text { at } x_{1}=0 \text { and } 1 .
$$

Equations (3)-(8), along with equations (1)-(16) in part 1 , completely define the problem of a freely deforming liquid pore.

To make the numerical investigations more relevant, we first ascertain the magnitude and sensitivity of the dimensionless groupings arising in (3), namely $\mathrm{Bo}, \mathrm{Vr}, \mathrm{Cr}$ and $\mathrm{Ca}$, over a range of thermophysical properties, pore widths $D$ and superheating/subcooling limits $|\Delta T|$. Based on the arguments given earlier in part 1 , we consider a range of $D$ from $10^{-2}$ to $10^{4} \mu \mathrm{m}$ and a range for $|\Delta T|$ of $10^{-1}$ to $10 \mathrm{~K}$. Plots of the upper and lower bounds for these parameters as a function of $D$ are shown in figure 2 .

$B o$ and $C a$ are independent of $|\Delta T|$ and exhibit nearly opposite functional dependence on $D$ (i.e. $B o \propto D^{2}$ and $C a \propto D^{-1}$ ). This implies the existence of a capillarydominant regime at small characteristic dimensions $(D \sim 1 \mu \mathrm{m})$ where $C a \gg B o$, and the velocity and dynamic pressure terms in (3) influence surface morphology. Depending on the value of $|\Delta T|, V r$ and $C r$ may also affect the geometry. Although $V r$ is proportional to $C a$, it also varies inversely with the square of $R s$ (where $R s \propto 1 / D$ ) which means that $V r \propto D$. At larger characteristic dimensions $\left(D \sim 10^{4} \mu \mathrm{m}\right), B o$ predominates, and we can effectively ignore contributions from dynamic pressure. Additionally, both $V r$ and $C r$ are small at low $|\Delta T|$, and here, too, we expect the meniscus to be governed entirely by hydrostatic pressure. However at higher superheating/subcooling levels, $(|\Delta T|=10 \mathrm{~K})$ contributions from both $V r$ and $C r$ can exceed hydrostatic effects. In summary, we see that $B o$ can be neglected for the size range of interest here. For calculation of the meniscus surface, $\mathrm{Cr}$ can range from $10^{-4}$ 
for the low value of superheat/subcooling to $10^{-1}$ at $1 \mathrm{~K}$. We also expect a contribution from $C a$ which varies from $10^{-5}$ to $10^{-2}$.

\section{Solution methodology}

Since a detailed description of the numerical model is given in part 1 , we will only summarize its key features in this section. Although it is possible to structure a numerical solution so that the surface is determined simultaneously with $V_{i}, P$ and $T$, this approach is memory-intensive and requires manipulation of an extremely large sparse coefficient matrix. An alternative approach, which was implemented by Cuvelier \& Driessen (1986) and Hyer, Jankowski \& Neitzel (1991), relaxes the normal-stress boundary condition and calculates surface position $y^{(s)}$ in an iteration loop outside the steady-state flow-field solution. In this 'trial method', the meniscus solution is the outermost iteration in a 5-loop flow-field solution procedure. The problem of accommodating small contact angles is handled by replacing $\kappa$ with $\partial \alpha / \partial s$ and transforming (3) into an integro-difference equation. The surface position $s$ and $\alpha$ can then be treated as independent and dependent variables, respectively. This approach was applied to purely hydrostatic configurations by Concus (1968) and Geiger (1966), and can be extended to include the effects of dynamic pressure, velocity and interfacial temperature. The resulting expression is

$$
\frac{\partial \alpha}{\partial s}=\frac{\Sigma-C a \Delta P+V r \Delta\left(T^{2}\right)+2 C a \Delta\left(V_{i, j}\right) n_{j} n_{i}+B o \int_{0}^{s} \sin \alpha \mathrm{d} s}{1-C r \bar{T}},
$$

where $\Sigma$ is the curvature at the meniscus centreline given by

$$
\Sigma=B o y_{c}^{(s)}+C a\left(P^{(v)}-P_{c}\right)+\frac{f_{p}-1}{R s^{2}} T_{c}^{2}+2 V_{c i, j} n_{j} n_{i} .
$$

Here, the subscript $c$ refers to the values of the variables at the meniscus centreline. Between the surface nodes at which flow-field data are available, $T, V_{i}$ and $P$ values are interpolated using the same order as their element-level variance. We have expressed (9) in a form where $\Sigma$ corresponds to the curvature at the meniscus centreline. Consequently, the curvature at other points along the surface is a function of $\Sigma$ and the change in pressure, temperature and velocity relative to the centreline values, that is $\Delta P=P-P_{c}, \Delta\left(T^{2}\right)=T^{2}-T_{c}^{2}$ and $\Delta\left(V_{i, j}\right)=V_{i, j}-V_{c i, j}$.

The entire surface extending from $x_{1}=0$ to 1 is solved by applying the shooting method to each half of the meniscus. At the centreline $x_{1}=0.5$, we set $\alpha=0$, assume a value of $\Sigma$ and calculate $\alpha$ at successive steps along the contour until the contact line is reached. If the final contact angle estimate fails to match, within a specified tolerance, the desired value of $\omega, \Sigma$ is adjusted, and the contour integration is repeated. The new estimate of $\Sigma$ is obtained using a simple bisection algorithm, which can accommodate large positive or negative values of $\Sigma$.

\section{Preliminary analysis}

Although the method outlined above can be used to obtain steady-state solutions, it is computationally too expensive for a complete parametric assessment. It is preferable to first perform a preliminary analysis which treats surface deformation as a second-order effect. In this approach, the velocity, pressure and temperature fields which we determined earlier with a fixed surface (part 1) are used to calculate meniscus 
shape via the integration procedure described in $\S 3$. We can also apply this method to estimate the contribution from each stress term in (3). For example, the influence of surface tension variation due to temperature change, which is embodied in the denominator of (3), is examined by varying $\mathrm{Cr}$ while holding the parameter coefficients of all the other terms equal to zero. This approach is valid for small deformations but fails when the deflections become large. If the analysis indicates significant deformation at values of $\mathrm{Ca}, \mathrm{Cr}$ and $\mathrm{Vr}$ within the identified scaling limits, then the complete solution must be considered.

In this section we estimate the changes in meniscus shape caused by each term in (3) and (8), namely surface tension, recoil force, pressure, viscous stress and contact angle. When examining deformation, it is important to note that all surface variables are fixed at the contact line. Thus, variations in surface morphology are due to the change of these variables relative to their contact line values.

\subsection{Surface tension dependence}

Surface tension variation along a non-isothermal meniscus contributes to deformation not only through the convection arising from thermocapillary stress but also through the adjustment of capillary pressure to changes in temperature. The latter effect is manifested by the $(1-C r \bar{T})^{-1}$ term in (3), where $C r$ represents the sensitivity of surface tension to temperature. Warmer regions of the surface assume larger curvatures $\kappa$ to offset the reduction in surface tension. The ratio of surface tension at the highest temperature $\gamma_{h}$ to that at the lowest temperature $\gamma_{l}$ is given by

$$
\gamma_{n} / \gamma_{l}=1-C r
$$

A limitation of the linear equation of state for $\gamma$ and the definition of $\mathrm{Cr}$ becomes obvious when $C r$ approaches 1 . In this case, the maximum temperature limit $T_{h}$ corresponds to the critical temperature, and with superheating $\gamma$ vanishes and $1 / \kappa \rightarrow \infty$ at the contact line. Although the parameter bounds obtained in $\$ 2$ indicate that the range of $\mathrm{Cr}$ is small enough to justify use of a linear approximation, meniscus shapes will nonetheless be determined for $\mathrm{Cr}$ values close to unity in order to identify the limiting values for strong deformations.

Surface-tension-induced deformation is first examined using the basic-state interfacial temperature distributions $(M a=0$ and $R s=\infty)$. Half-cavity meniscus contours for $B i=1,10$ and $10^{2}$, and $C r=0.5,0.9,0.99$ and 0.999 are shown in figure 3 . The responses to the two heating modes are opposite. With subcooling, the temperature at the centreline is a maximum and decreases to the minimum $T_{l}$ at the sidewalls. Because of the lower surface tension at the centre, the surface assumes a higher curvature there relative to the sidewall region. At low $B i$, the effect is small, even at high $\mathrm{Cr}$, because temperature remains close to the minimum limit along the entire surface. At high $B i$, the temperature in the centre approaches the maximum and the surface becomes more sensitive to $C r$. Although the deformation appears to be quite large at high $B i$, it is still negligible for the more realistic values of $C r<0.5$. With superheated boundaries, temperature decreases towards the centreline, where the curvature assumes its minimum value. The result is a flattening and raising of the meniscus with respect to the contact line. At low $B i$, the temperature remains close to the high sidewall value along the entire surface and is more sensitive to variations in $\mathrm{Cr}$. At higher $\mathrm{Bi}$, however, the lower temperature at the centre yields a surface that is less sensitive to $C r$.

The influence of fluid convection on surface-tension-induced deformation reflects the departure from the basic-state interfacial temperature distribution. Evaporation and 

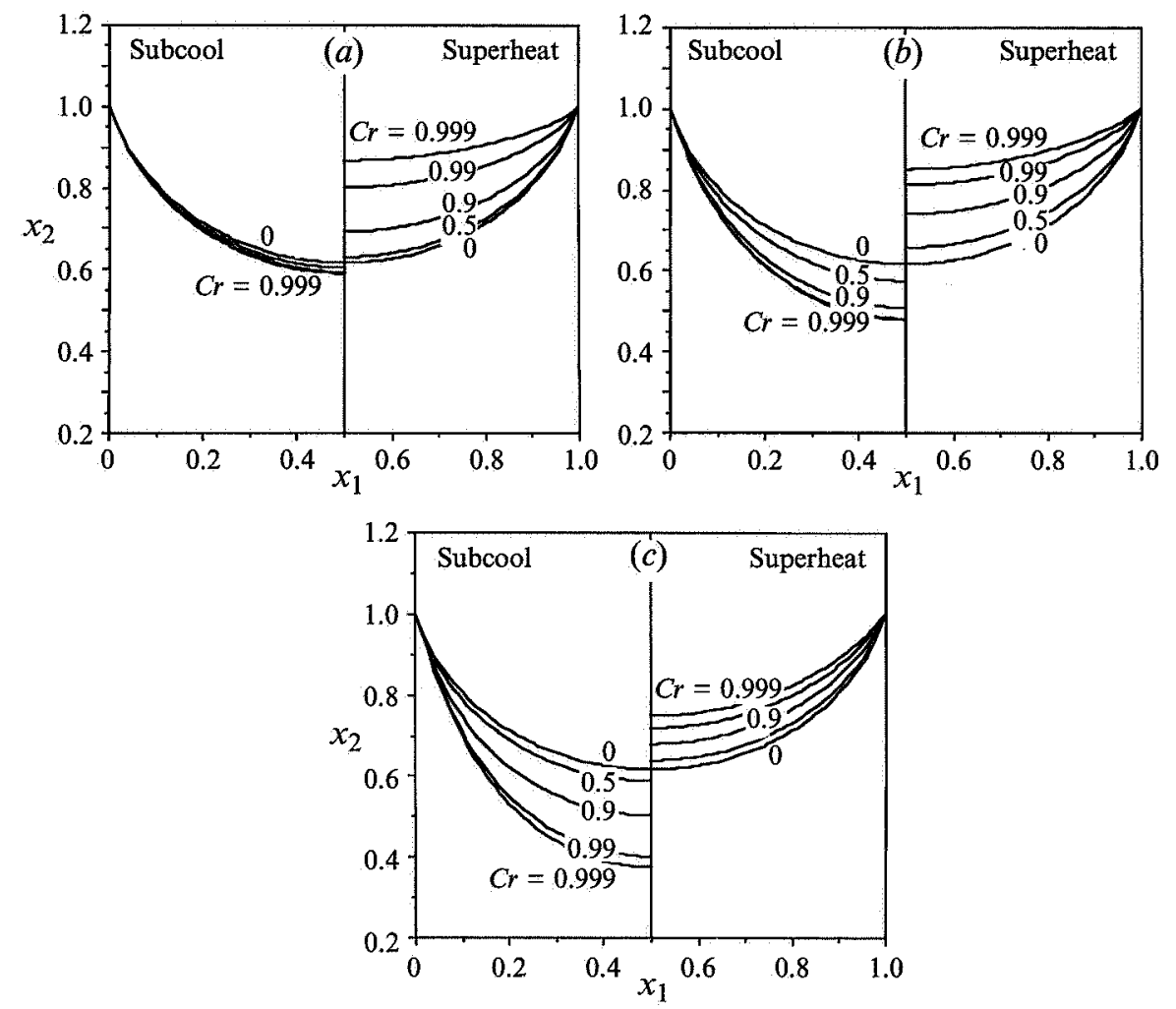

FIGURE 3. Surface-tension-induced deformation based on basic-state interfacial temperature distribution for $\omega=15^{\circ}$ : half-cavity meniscus contours for (a) $B i=0,(b) B i=10,(c) B i=100$.

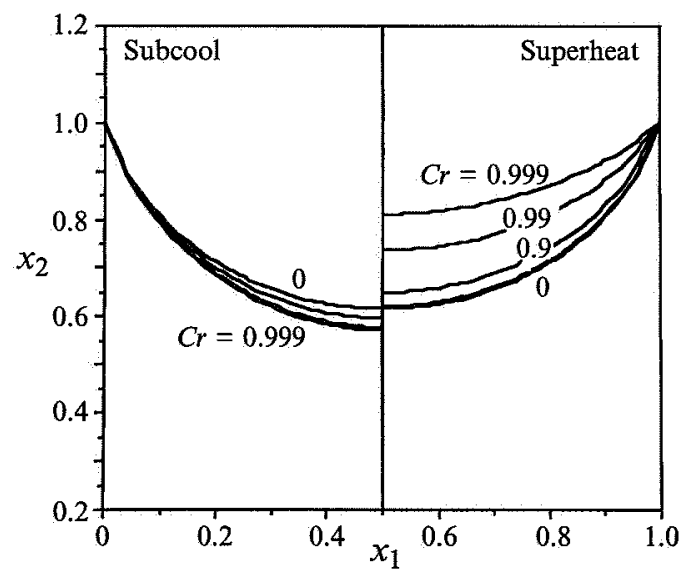

FIGURE 4. Surface-tension-induced deformation based on surface temperature distribution for pure interfacial flow, where $M a=0, R s=10^{-1}, B i=1$ and $\omega=15^{\circ}$.

condensation both increase the average temperature of the interface. This reduces the average temperature gradient for evaporation but increases it for condensation. The effect of pure interfacial flow on surface geometry is shown in figure 4 for $B i=1$ and $R s=10^{-1}$. Although the differences are quite small, condensation appears to increase deformation relative to the basic state, while evaporation decreases it. The surface is 


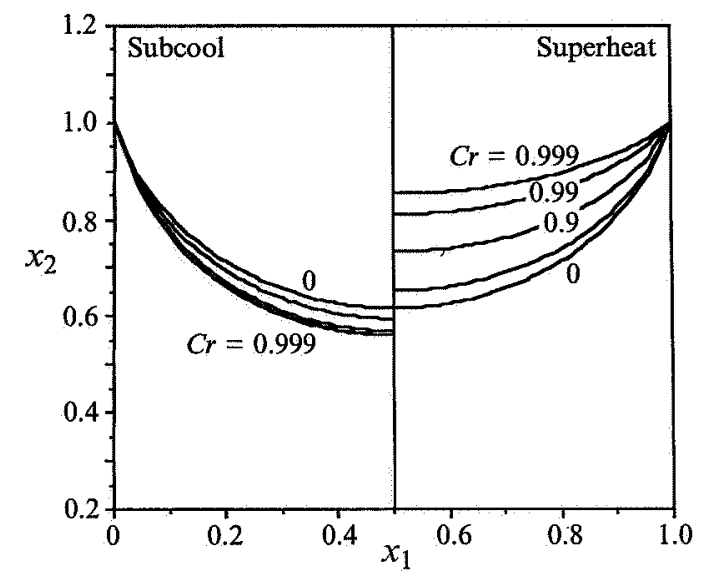

FIGURE 5. Surface-tension-induced deformation based on surface temperature distribution for pure thermocapillary flow, where $M a=10^{3}, R s=10^{3}, B i=10$ and $\omega=15^{\circ}$.

also more sensitive to $\mathrm{Cr}$ for both heating modes because of the increase in average temperature. The sensitivity of surface morphology to pure thermocapillary flow with $M a=10^{3}$ and $B i=10$ is shown in figure 5. Unlike the previous cases, the thermocapillary convection associated with subcooling tends to lower interfacial temperature relative to the basic state, while superheating raises it. Consequently, the subcooling regime exhibits less sensitivity to $C r$ than its basic-state counterpart in figure 3. The deformation associated with superheating, however, behaves very similarly to the basic state at $B i=10$. In all these cases, the effect of surface tension appears to be negligible for $\mathrm{Cr}$ less than 0.5 , and can safely be ignored in this problem.

\subsection{Vapour recoil dependence}

The recoil force is proportional to the square of the surface mass flux and temperature difference between the meniscus and bulk vapour, which translates to a $T^{2}$ dependence. To examine its influence, we employ, as before, the basic-state temperature distribution as an initial reference. Although this regime lacks the effect of internal convection, it does illustrate the fundamental response to changes in thermal non-equilibrium. Figure 6 shows the meniscus shapes for $B i=1,10$ and $10^{2}$. Since the temperature magnitudes for superheating and subcooling are equivalent, the recoil force distribution for the basic state is independent of heating mode. The contribution of recoil to surface curvature is highest near the contact line because the temperature difference and mass flux are a maximum there. That is, the curvature must be higher near the sidewalls to compensate for the larger normal force directed into the liquid. Over the entire range of $B i$, raising $V r$ magnifies the variation in curvature and tends to flatten the surface relative to the contact line. Unlike the influence of $\mathrm{Cr}$, the curvature dependence on $\mathrm{Vr}$ is strong, and at very high $V r$ the centreline curvature can become negative and form an inflection along the meniscus.

It is evident from figure 6 that the sensitivity to $B i$ is maximized between 1 and $10^{2}$ owing to the shift in temperature gradient towards the sidewall with increasing $B i$. As $B i$ is lowered, the scaled temperature along the meniscus approaches the isothermal sidewall value. Thus, the surface temperature difference and curvature are relatively constant, and deformation is minimized. As $B i$ is increased, the variation of the centreline meniscus temperature from the sidewall value is higher, and causes a corresponding increase in surface deflection. However, at very high values of $B i$, the 

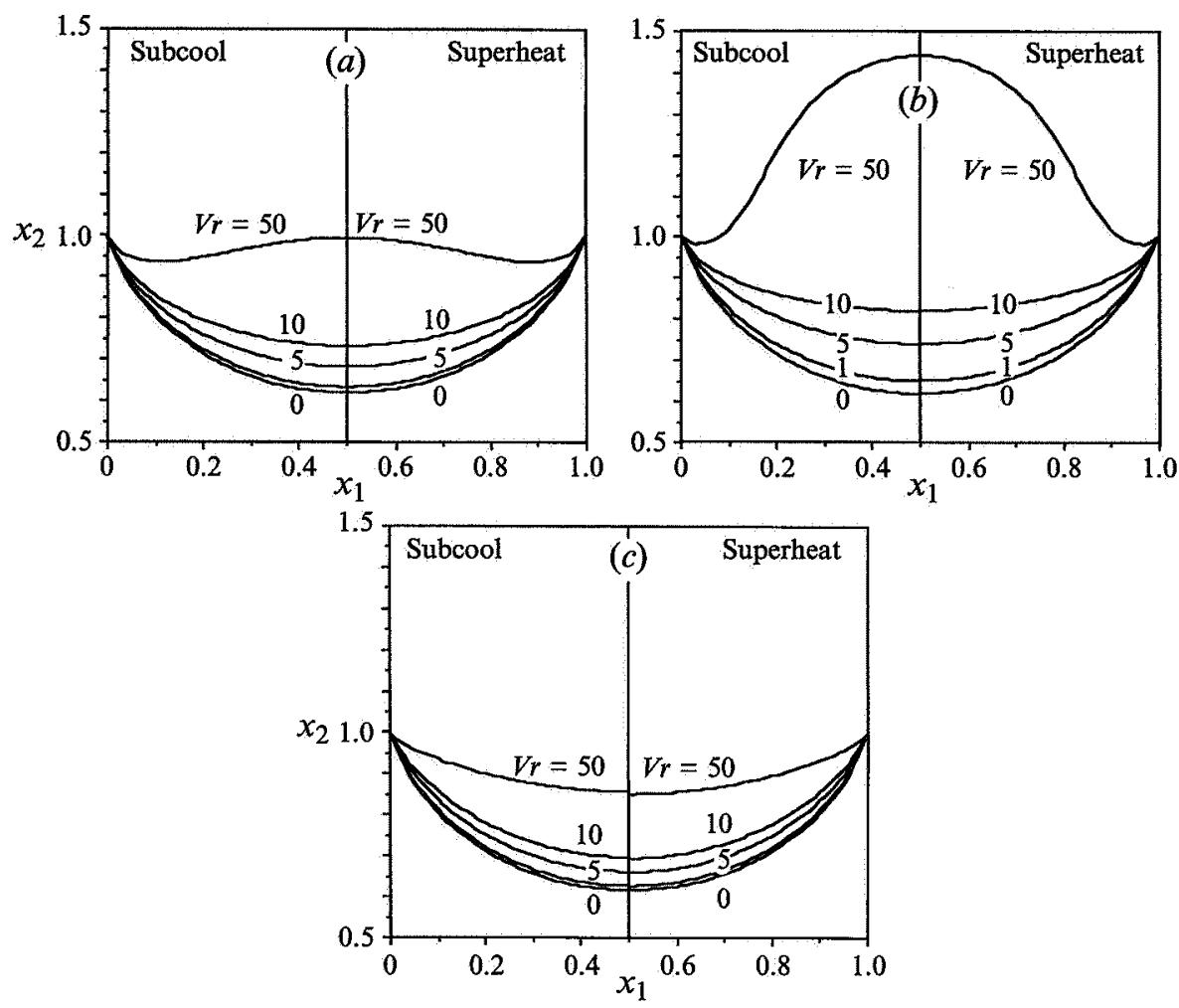

FIGURE 6. Vapour-recoil-induced deformation based on basic-state interfacial temperature distribution for $\omega=15^{\circ}:(a) B i=0,(b) B i=10,(c) B i=100$.

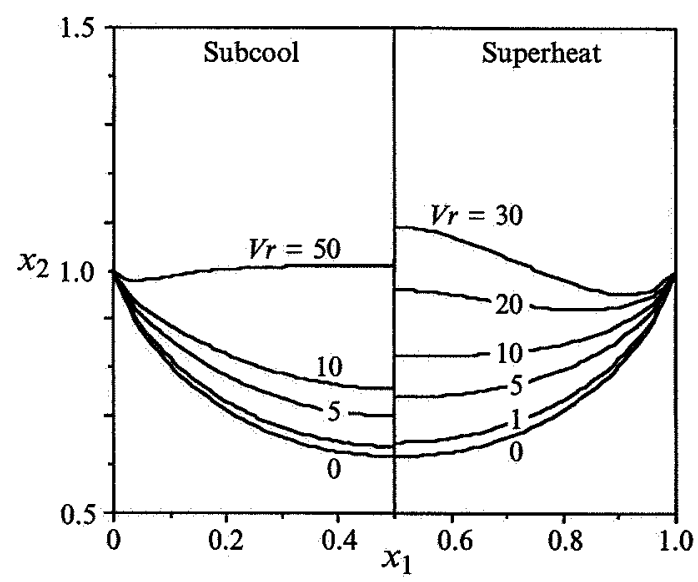

FIGURE 7. Vapour-recoil-induced deformation based on surface temperature distribution for pure thermocapillary flow, where $M a=10^{3}, R s=10^{3}, B i=10$ and $\omega=15^{\circ}$.

interfacial distribution approaches the bulk vapour temperature followed by a sharp increase or decrease to the sidewall value near the contact line. Because the influence of this sharp change on overall curvature is slight, the deformation diminishes with increased $B i$ after attaining its peak value.

The influence of pure thermocapillary flow $\left(M a=10^{3}\right.$ and $\left.B i=10\right)$ is illustrated in figure 7 . Although the convective influence of phase change is not present here, the 


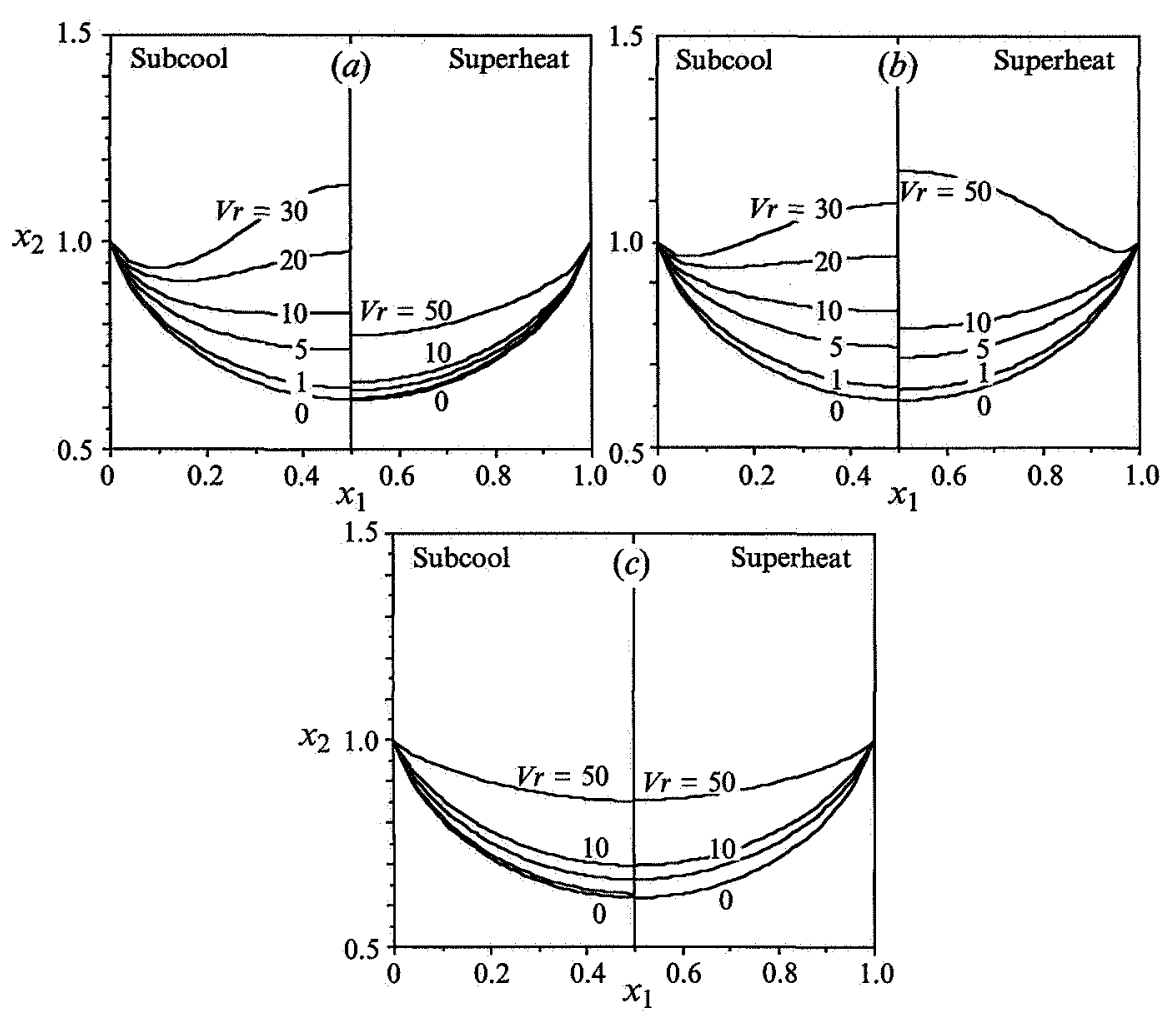

FIGURE 8. Vapour-recoil-induced deformation based on surface temperature distribution for pure interfacial flow, where $M a=0, R s=10^{-1}$ and $\omega=15^{\circ}:(a) B i=1,(b) B i=10,(c) B i=100$.

temperature distribution adequately reflects the effect of thermocapillary convection. With subcooling, thermocapillary flow shifts the temperature gradient towards the sidewall and causes an upwelling of cool liquid that flattens the thermal distribution around the centre of the cavity. This is similar to increasing $B i$ in that the meniscus becomes less sensitive to recoil. The deformation here is less than in the corresponding basic state, with the maximum deformation occurring at a lower value of $B i$. Although it is not shown here, varying $B i$ causes deformation similar to the response based on the basic state in figure 6. Superheating, which acts opposite to subcooling, counters the tendency of the surface temperature distribution to flatten with increased $B i$. Thus, the maximum deformation occurs at higher $B i$. As with subcooling, the deflection decreases at very high and low $B i$.

The sensitivity of surface morphology to pure condensation and evaporation ( $R s=10^{-1}$ and $B i=10$ ) is shown in figure 8. Condensation accumulates warm liquid around the centreline and increases the surface temperature gradient, while evaporation flattens the temperature distribution. Compared with the basic state, condensation produces a maximum deformation at lower values of $B i$, while evaporation does so at higher values of $B i$. Surface deflection again diminishes in the limit of very high and low $B i$.

\subsection{Dynamic pressure dependence}

Our analysis in part 1 showed that fluid motion can yield substantial pressure variations along the free surface. The influence of the dynamic pressure on surface morphology is manifested by the $\mathrm{CaP}$ term in (3) and is shown for pure thermocapillary 

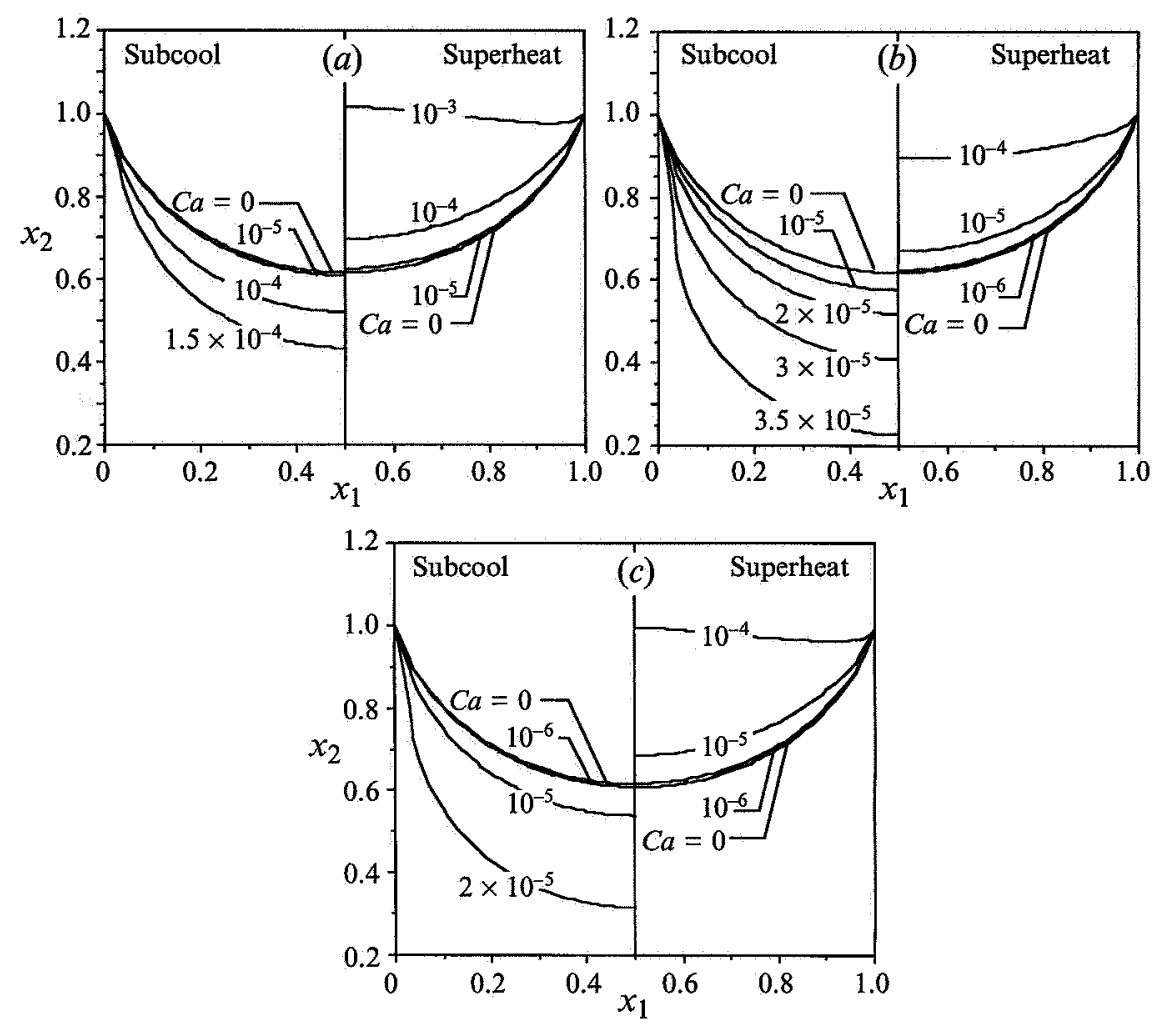

FIGURE 9. Pressure-induced deformation based on surface temperature distribution for pure thermocapillary flow, where $M a=10^{3}, R s=10^{3}$ and $\omega=15^{\circ}:$ (a) $B i=1$, (b) $B i=10$, (c) $B i=100$.

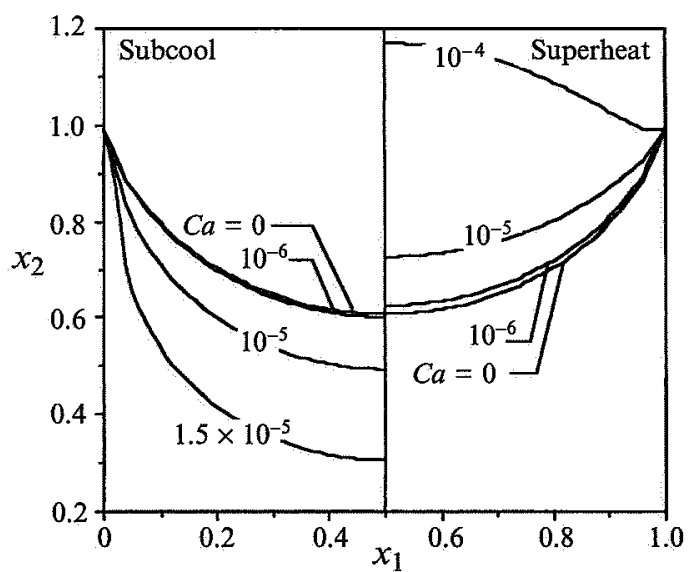

FIGURE 10. Pressure-induced deformation based on surface temperature distribution for pure combined thermocapillary/interfacial flow, where $M a=10^{3}, R s=10^{-1}, B i=10^{2}$ and $\omega=15^{\circ}$.

flow $\left(M a=10^{3}\right.$ and $\left.R s=10^{3}\right)$ in figure 9 . The capillary number $C a$ is a measure of the magnitude of the reference surface tension in that higher values of $C a$ correspond to lower $\gamma$. Since surface tension acts to minimize the interfacial area and deformation, the deflection becomes greater with larger values of $\mathrm{Ca}$. The increase in deformation 
with $B i$ is consistent with the substantial increase in interfacial pressure gradient noted in our previous study. In part 1 it was shown that subcooling causes the pressure to drop sharply from the sidewalls resulting in a suction in the middle of the cavity. It is apparent in figure 9 that this suction pulls the surface down in the centre. Superheating, however, produces a pressure increase in the centreline relative to the sidewalls, which raises the meniscus relative to the undeformed state. The trends are the same as that for subcooling in that higher values of $B i$ and $C a$ increase deformation.

Our earlier analysis also showed that the surface pressure gradient rises substantially when thermocapillary flow is combined with evaporation or condensation. This should cause a corresponding increase in surface deformation. The steady-state surface calculations shown for the combined flow case $\left(M a=10^{3}, R s=10^{-1}\right.$ and $\left.B i=10^{2}\right)$ in figure 10 confirm that the deformation does indeed increase. It is clear from figures 9 and 10 that, as anticipated from part 1 , the interfacial distribution of dynamic pressure strongly affects surface morphology.

\subsection{Viscous stress dependence}

The effect of viscous stress on surface morphology is represented by the $2 \mathrm{Ca} V_{i, j} n_{j} n_{i}$ term in (3). With the integral solution methodology, it is preferable to transform the term into an expression that does not involve the velocity gradient. Application of the chain rule and the scaled equations for mass flux and surface heat transfer yield

$$
V_{i, j} n_{j} n_{i}=-S T-V_{i} n_{j} n_{i, j}
$$

where $S=B i / R s . S T$ is termed the 'flux component' and represents stress arising from flow normal to the surface. $V_{i} n_{j} n_{i, j}$ is the so-called 'curvature component'. With a nonvolatile surface, $1 / R s=0$ and $S$ vanishes, while with a flat surface, $n_{i}$ is constant and $n_{i, j}=0$. The curvature component can also vanish if the tangential velocity is negligibly small.

We first examine the response using the basic-state temperature distribution as a reference. Since there is no internal convection associated with this regime, only the influence of $S T$ can be examined. Figure 11 shows the half-cavity surfaces with subcooled and superheated boundaries for $B i=1,10$ and $10^{2}$ where the sensitivity is characterized in terms of $C a S$. Unlike recoil, which is proportional to the square of temperature, the flux term can assume negative and positive deviations, depending on the temperature gradient relative to the centreline. Subcooling produces positive temperature differences and mass fluxes similar to the recoil force variation. The surface deformation consequently resembles the response to the recoil parameter. Superheating, on the other hand, results in a negative temperature difference between the meniscus and the bulk vapour and causes a depression about the centreline.

Increasing the product $C a S$ is equivalent to decreasing either the surface tension $\gamma$ or interfacial resistance $R s$. Figure 11 clearly shows that both of these trends magnify meniscus deformation. The effect of $B i$ on the flux component, by modifying the surface temperature distribution, is similar to that of the recoil parameter and dynamic pressure. Figure 11 also shows that the deflection undergoes a maximum at an intermediate $B i$ and vanishes at the limits of very low and high $B i$.

Although vapour recoil produces greater deformation than this case, due to its quadratic dependence on the temperature difference, the viscous-dependent deflection based on the basic-state temperature is nonetheless substantial. This seems to indicate that viscous stress contributions to the interfacial curvature are significant. However, when the more meaningful cases of pure evaporation/condensation and pure thermocapillary flow are considered, the results are quite different. Figure 12 illustrates 
Thermocapillary flow at low gravity. Part 2
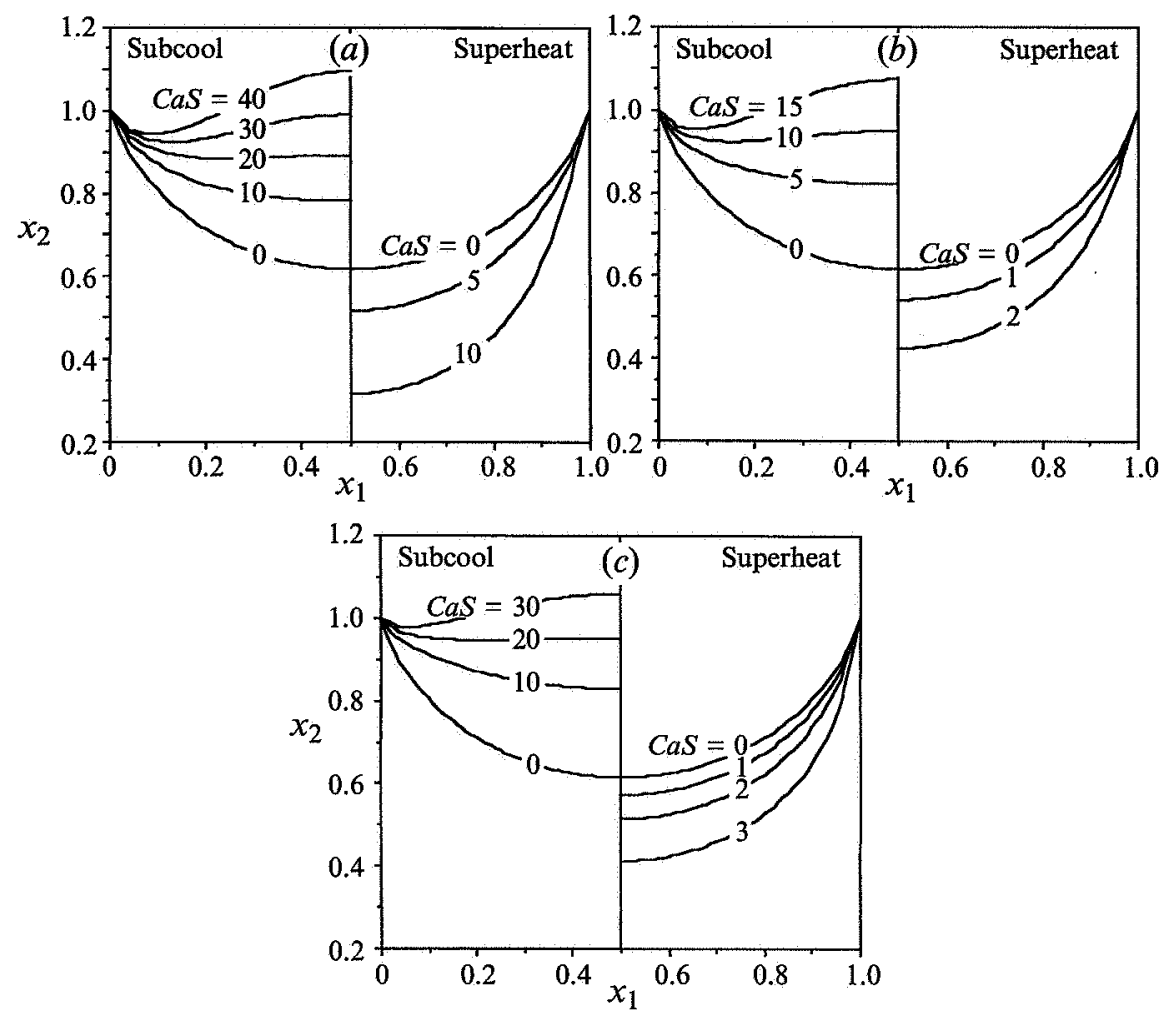

FIGURE 11. Viscous-stress-induced deformation based on basic-state interfacial temperature distribution for $\omega=15^{\circ}:(a) B i=1,(b) B i=10,(c) B i=100$.

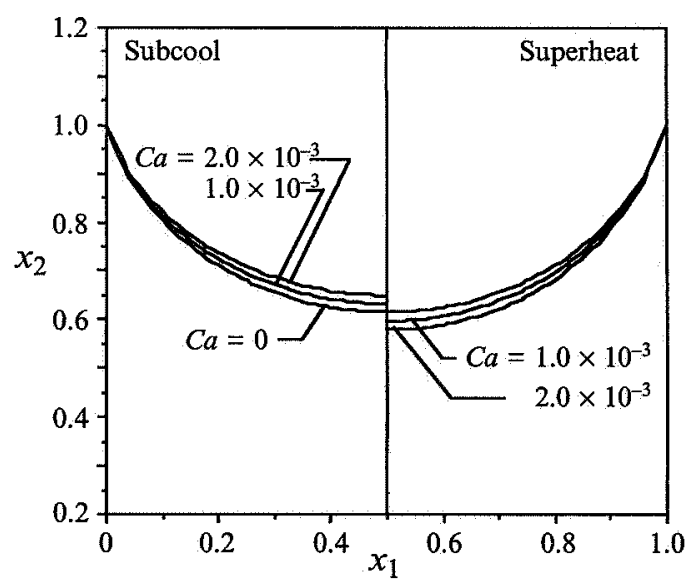

FIGURE 12. Viscous-stress-induced deformation based on surface temperature distribution for pure interfacial flow, where $M a=0, R s=10^{-1}, B i=10$ and $\omega=15^{\circ}$. 


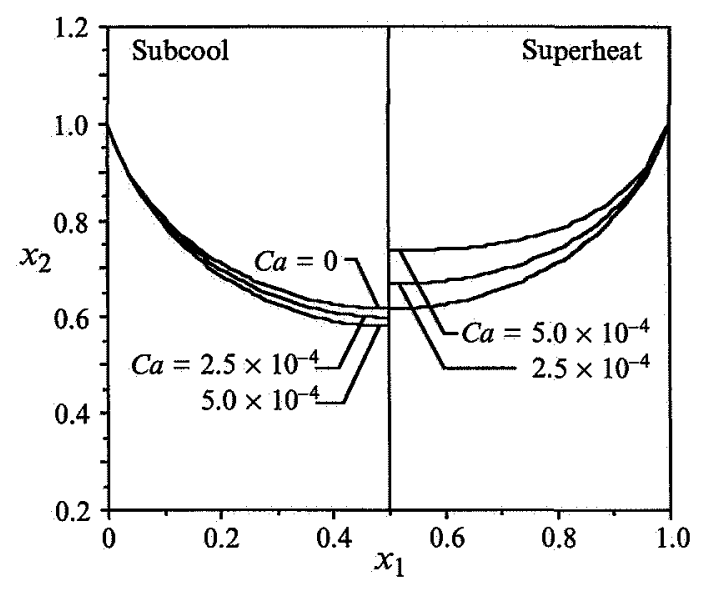

FIGURE 13. Viscous-stress-induced deformation based on surface temperature distribution for pure thermocapillary flow, where $M a=10^{3}, R s=10^{3}, B i=10$ and $\omega=15^{\circ}$.

the influence of pure evaporation and condensation (with $R s=10^{-1}$ and $B i=10$ ), and figure 13 shows the results for pure thermocapillary flow $\left(M a=10^{3}\right.$ and $\left.B i=10\right)$. The viscous stress contributions are small for both cases and can be safely neglected.

\subsection{Contact angle dependence}

When considering the isothermal static solution, the only effect of contact angle is a variation in centreline curvature. That is, lower contact angles translate to an increase in curvature along the entire meniscus. With deformation, however, the influence of contact angle must be viewed within the context of convection in the cavity since in part 1 it was shown that the flow field is also extremely sensitive to $\omega$. The primary effects of lowering the contact angle are an increase in the net thermocapillary stress force, half-cavity circulation and interfacial pressure gradient.

With subcooling, the positive pressure gradient towards the sidewall is amplified with reduction of contact angle. This applies even when $C a$ and all other parameters are held constant. The effect of this tends to magnify the depression from the isothermal static reference, and is further accentuated by the non-convective increase in centreline curvature associated with reduced contact angle. With superheating, the pressure gradient is also increased by reducing the contact angle. However, since the deformation is positive relative to the contact line, this tends to counter the centreline curvature associated with the isothermal static solution. It should be clear that the firstorder influence of contact angle on deformation reflects the convection-induced dependence on dynamic pressure and the changes in the static solution, and has a significant impact on the meniscus shape.

\section{Complete analysis}

The preliminary analysis indicated that surface morphology is most sensitive to the flow-induced dynamic pressure, the recoil force arising from interfacial mass transfer and the contact angle. It was also shown that recoil always acts to raise the meniscus, while dynamic pressure tends to lower it for subcooling and raise it for superheating. In this section, we investigate the simultaneous influence of dynamic pressure and vapour recoil on morphology in response to changes in liquid/vapour equilibrium. The 

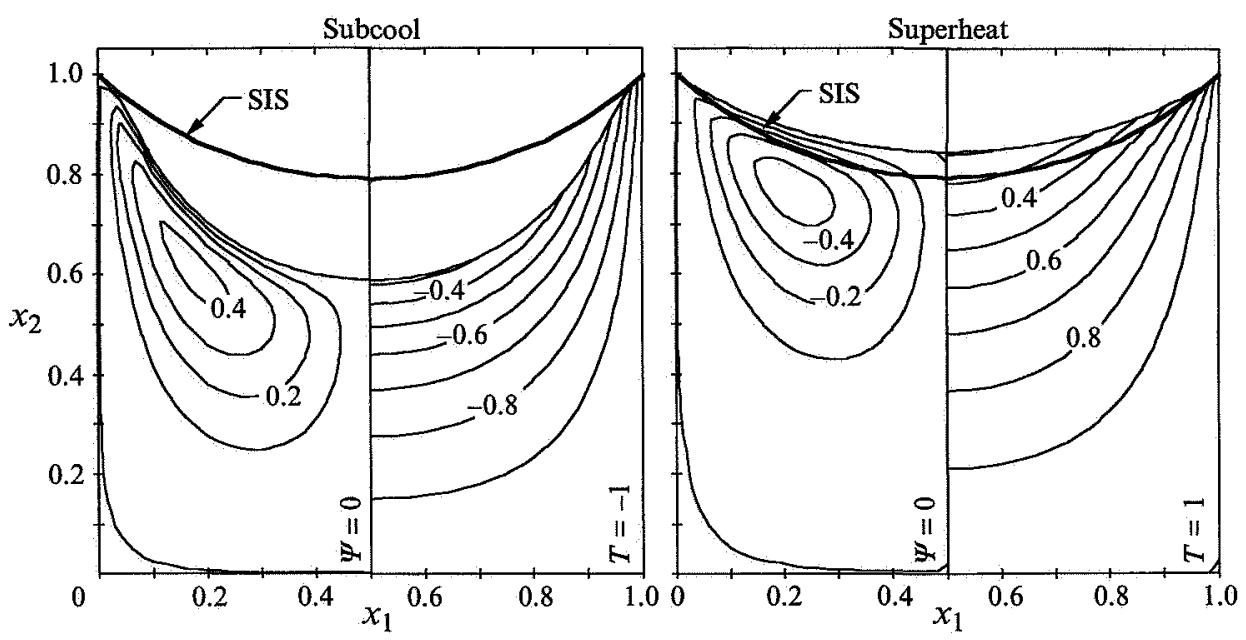

FIGURE 14. Steady-state flow field/surface solution for $M a=10^{2}, R s=10^{3}, B i=10, C r=10^{-1}$ and $\omega=15^{\circ}$. SIS denotes static isothermal solution.

objective is to evaluate the surface's ability to maintain mechanical equilibrium by determining whether solutions exist that simultaneously satisfy the equations of fluid motion, temperature and surface geometry.

Within the context of the one-sided model, the only parameters that directly reflect a change in vapour/liquid equilibrium are $R s$ and $B i$, where $V r \propto R s^{-2}$. All others, except $\omega$, are fixed at values approximately representative of a 1 to $10 \mu \mathrm{m}$ pore subjected to superheating/subcooling levels of $10^{-1}$ to $1 \mathrm{~K}$, namely $M a=10^{2}$, $C r=10^{-1}, C a=10^{-3}$ and $f_{\rho}=10^{3}$. We first examine the influence of interfacial equilibrium while holding heat transfer characteristics and all other dimensionless groupings constant. $R s$ and $\operatorname{Vr}\left(\propto R s^{-2}\right)$ are varied to model different levels of vapour/liquid equilibrium along the surface. The sensitivity of latent heat transport to mass transfer is ignored by holding $B i$ constant at 10 , which is the value near which recoil is maximized. We begin by focusing on thermocapillary effects by considering the approximately non-volatile case in which $R s=10^{3}$. After that, we examine progressively lower values of $R s$ (and higher $V r$ ) to determine how extensively recoil either augments or offsets the influence of dynamic pressure. The contact angle $\omega$ is also varied from $15^{\circ}$ to $45^{\circ}$ to model the effect of various wetting fluids.

We attempted to obtain a solution for the non-volatile case of $R s=10^{3}, M a=10^{2}$ and $\omega=15^{\circ}$. However, with subcooling, the large circulation intensities yielded excessive dynamic pressure gradients along the surface near the contact line, and it was impossible to obtain convergence between the flow field and surface solutions. Similar behaviour was observed with an even larger contact angle of $45^{\circ}$. This is indicated in figure 14 which shows the flow fields for this case superimposed on the static isothermal solution (SIS). With subcooling the surface diverges to a multivalued shape, and we are unable to obtain convergence between the flow field and surface. The subcooling case in figure 14 actually represents the steady-state solution after the 16th surface iteration. With subsequent iterations, the depression near the centreline grows until the surface becomes multivalued at $x_{1} \approx 0.1$ and 0.9 . The same instability was encountered with even finer element grids. The divergence indicates that $P$ is strongly influenced by the contour and growing depression with each surface iteration. During the first few iterations, the meniscus assumes an inflection to accommodate the negative 

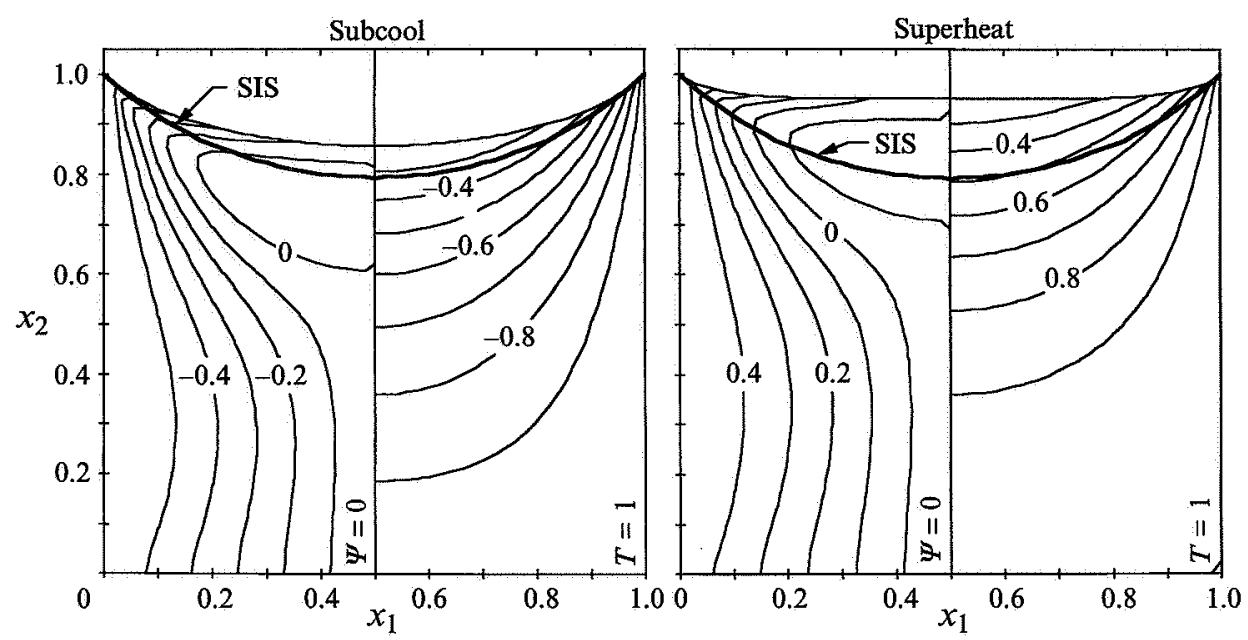

FIGURE 15. Steady-state flow field/surface solution for $M a=10^{2}, R s=1 / \sqrt{ } 10, B i=10, C r=10^{-1}$ and $\omega=15^{\circ}$.

contribution of $P$ to the contour angle integration. The flat surface around this inflection extends the high-pressure region into the centre, which drives the depression even lower. Another contributing effect is the increase in circulation intensity with each iteration pass, which is greater than for the fixed-shape counterpart studied in part 1. The depressed surface around the centreline serves to increase the net thermocapillary stress force and promotes circulation. Consequently, the deformation associated with subcooling has the same effect on $P$ as reducing contact angle. It is clear that subcooling can lead to excessive surface deformations, especially with highly wetting fluids.

With superheating, a stable steady-state solution was obtained after only eight surface iterations. As this state is approached, the meniscus flattens and decreases in area due to the pressure drop towards the wall. This tends to reduce the half-cavity circulation relative to its non-deforming counterpart. The deformation here models the effect of increasing contact angle which serves to reduce the interline pressure gradient and ensures stable numerical convergence. As long as the surface retains a positive curvature, superheated thermocapillary flow should promote mechanical equilibrium along the surface.

To test whether vapour recoil could reduce or possibly eliminate the pressure-driven instability arising from subcooled thermocapillary flow, we investigated the case of $\omega=45^{\circ}, R s=1 / \sqrt{ } 10$ and $V r=10$ (figure 15 ). The solution for the subcooling mode is completely stable and slightly raised with respect to the static isothermal solution curve. The order of magnitude increase in $V r$ is enough to completely offset the influence of dynamic pressure. With superheating, the vapour recoil and dynamic pressure both act to raise the meniscus, and deformation from the static isothermal state is much greater. We also tested the limiting large value of $R s$ for $\omega=45^{\circ}$ beyond which the surface becomes multivalued for subcooling. For $R s=1 / \sqrt{ } 5$ we obtained a surface that projects slightly below the static isothermal solution contour, while for all values of $R s>1 / \sqrt{ } 5$, the solution becomes multivalued and the iteration diverges. Note that smaller contact angles require lower $R s$ values to ensure convergence and a stable solution.

The pressure and recoil-related deformation resulting from superheating causes an 


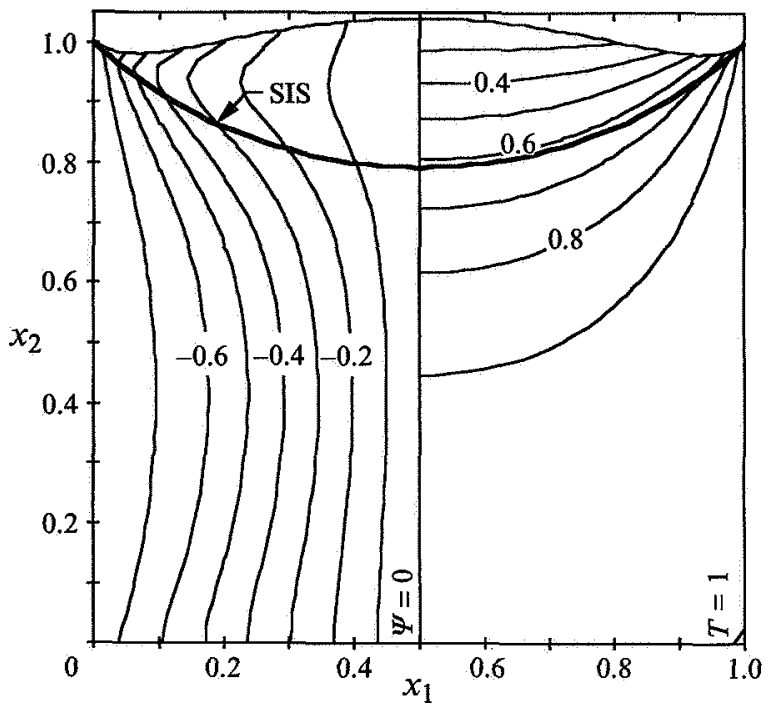

FrgURE 16. Steady-state flow field/surface solution for superheating with $M a=10^{2}, R s=1 / \sqrt{ } 20$, $B i=10, C r=10^{-1}$ and $\omega=15^{\circ}$.

upward-pointing bulge in the middle of the meniscus. Since the surface in figure 15 is nearly flat, it is probable that a reduction in $R s$ from the specified value of $1 / \sqrt{ } 10$ should yield an inflection and negative curvature at the centreline. We test this for $\omega=45^{\circ}$ by reducing $R s$ to $1 / \sqrt{ } 20$ and doubling $V r$ from 10 to 20 . The resulting steadystate flow field in figure 16 indicates that a stable solution can be obtained with a substantial negative curvature at the centreline. It is clear that reducing $R s$ further will cause the surface to become multivalued, first for superheating and then for subcooling.

Although we did not perform any systematic variation of $M a$, the outcome of such an analysis is easy to predict. Increasing $M a$ results in increased circulation intensities from thermocapillary flow. This in turn produces large meniscus dynamic pressure gradients, depressing the meniscus further for subcooling and raising it for superheating. Larger values of $\mathrm{Ca}$ further magnify this effect.

In summary, we see that vapour recoil always acts to flatten the meniscus, while thermocapillary flow raises and depresses it for superheating and subcooling, respectively. These trends are accentuated with larger $\mathrm{Ca}$ and $\mathrm{Bi}$. Reducing contact angle not only depresses the static isothermal solution contour but also enhances the influence of thermocapillary flow by increasing circulation intensity. A combination of these effects can easily lead to excessive meniscus rise or depression and possible loss of mechanical equilibrium. Because meniscus depression tends to reinforce itself by further increasing the net thermocapillary stress force and circulation, highly wetting fluids subjected to subcooling are more prone to this instability mechanism.

\section{Conclusions}

The influence of two-phase thermocapillary convection on the free surface behaviour of a volatile wetting liquid in a small open cavity has been examined. Owing to the significant computational requirements for simultaneous solution of the flow field and surface, we initially estimated the first-order contributions of various normal stress terms in the governing equation for surface curvature. This analysis showed that the 
influence of thermally induced surface tension variation and viscous stress is practically negligible. It was also evident that the deformation associated with the flux and curvature components of viscous stress exhibit opposite behaviour and tend to counteract each other in the case of combined convection.

The preliminary analysis revealed that the most important terms are vapour recoil, dynamic pressure and contact angle. In general, the influence of recoil is independent of heating mode and always acts to raise the meniscus. At high $V r$, resulting from low $R s$, the centreline curvature can become negative, and, if $V r$ is large enough, lead to a multivalued surface. The influence of dynamic pressure on surface geometry is quite different for subcooling and superheating. The pressure distribution associated with subcooling produces a suction in the middle of the meniscus that draws the surface down about the centreline. The distribution for superheating, however, is opposite and causes an upward bulging about the centreline. The magnitudes of these deflections increase as $B i \rightarrow \infty$ and /or $M a \rightarrow \infty$. In combined flow, condensation and evaporation both augment the interfacial pressure gradients for either heating mode and enhance these deformations further. Additionally, increasing $\mathrm{Ca}$ is equivalent to decreasing the surface tension and was found to enhance deformations as well. The contact angle affects the surface by altering the static solution shape and half-cavity circulation. Lowering the contact angle leads to larger dynamic pressure gradients near the sidewall and correspondingly greater deformation.

These trends were confirmed in the complete analysis which accounted for simultaneous solution of the flow field and surface. Increasing thermocapillary flow tends to depress the interface for subcooling and raise it for superheating, while increased recoil resulting from interfacial phase change always acts to raise the surface. For subcooling, these two effects tend to cancel each other and suppress deformation. It was difficult to obtain stable solutions for highly wetting fluids for subcooling due to excessive meniscus depression. A relatively high contact angle of $\omega=45^{\circ}$ and substantial condensation flow at $R s \leqslant 1 / \sqrt{ } 5$ was required to obtain a stable solution. These results indicate that the menisci of highly wetting fluids can become unstable with subcooling, and that liquid/vapour conditions must be carefully selected to maintain mechanical equilibrium and pressure differences across porous surfaces.

\section{REFERENCES}

Concus, P. 1968 Static menisci in a vertical right circular cylinder. J. Fluid Mech. 34, 481-495.

Cuvelier, C. \& Driessen, J. M. 1986 Thermocapillary free boundaries in crystal growth. J. Fluid Mech. 169, 1-26.

GEIGER, F. 1966 Hydrostatics of a fluid in a cylindrical tank at low Bond Numbers. Brown Engineering Research Labs, Tech. Note R-207, July 1966.

HYER, J., JANKOWSKI, D. \& NeITZEL, G. 1991 Thermocapillary convection in a model float zone. AIAA J. Thermo. Heat Transfer 5, 577-582.

Schmidt, G. R., Chung, T. J. \& NadarajaH, A. 1995 Thermocapillary flow with evaporation and condensation at low-gravity. Part 1. Non-deforming surface. J. Fluid Mech. 294, 323-347. 